\title{
Implementation and operational investigations of bipolar gate drivers
}

\author{
Jean-Christophe CREBIER*, Manh Hung TRAN*, Jean BARBAROUX*, Pierre-Olivier JEANNIN* \\ *Grenoble Institute of Technology, Grenoble Electrical Engineering Lab (G2ELab), \\ ENSE3 - BP 46 - 38402 Saint-Martin-d'Hères Cedex, FRANCE \\ Email: crebier@g2elab.grenoble-inp.fr, tranm@g2elab.grenoble-inp.fr
}

\begin{abstract}
This paper deals with the investigation of simple implementation and design of bipolar gate driver for high side power transistor control. Bipolar gate signals are usually preferred for high switching dynamic control and power device shielding. Nevertheless, bipolar supplies are harder to implement and rely on numerous components that may result in significant reduction of overall converter robustness. An alternative solution is to use unipolar supplies which are more complex but integrable with specific gate driver circuits. The addition of resonant structure gives the possibilities for involving the efficiency of the gate driver and reducing further gate driver supply requirements. The paper presents theses issues based on several gate drivers and their supplies.
\end{abstract}

\section{INTRODUCTION}

Gate drivers for power devices such as IGBTs or MOSFETs are increasingly asked to offer high performances while being simple to implement and highly reliable. In order to provide operational flexibility, security and effective dynamic control, gate drivers are usually implemented with bipolar supplies. By using bipolar gate driver signals, charge and discharge sequences can be well controlled while ensuring optimal ON state polarization and effective natural shielding during the OFF state. Besides, a number of studies are engaged toward the operational optimization and the integration of the gate drivers in order to simplify their implementations and to maximize their performances [1][2]. In particular, it can be noticed that many research results have been published with attempt to reduce the power consumption of the drivers for very high switching frequency applications [3][4]. More specifically, resonant converters are designed to drive each power device in a converter trying to exchange energy between the sources and the gate rather than dissipating periodically the energy fed to the gate. However, the practical implementation of floating bipolar sources to power supply the gate drivers of high side transistors remain difficult to minimize and to integrate. Researches have been carried out in order to identify simple and cost effective solutions but they are not always suitable for effective operation of modern gate drivers [5][6][7]. Other researches have been carried out trying to integrate gate driver supplies in order to simplify their implementation and to minimize the impact of common mode currents [8][9][10]. Today, it remains difficult to implement high efficiency resonant or bipolar gate drivers with simple and integrable gate driver supplies. As a result, we decided to investigate the implementation and the operation of bipolar gate drivers with respect to their power supplies in order to identify what could be the best available solutions.

The following sections of the paper are dedicated to study and to analyze the operational combination of the gate driver and its isolated power supply especially for driving high side transistors where electrical isolation is required. The first part introduces the state of the art concerning the gate driver and the gate driver supplies. This is studied considering power converter topologies based on the implantation of regular high side n-type transistors for medium and high voltage $(100 \mathrm{~V}$ up to $600 \mathrm{~V})$ application and low to medium power( 10 to $10 \mathrm{~kW})$. A similar study will also consider the case of CMOS and derived converter topologies [11][12] for low to medium voltages (below $15 \mathrm{~V}$ and around $100 \mathrm{~V}$ ) and low to medium power levels (1 to $100 \mathrm{~W})$. The following section focuses on the combination of the bootstrap driver supply and specific gate driver topologies in order to perform high efficiency bipolar control signals. The last section offers a comparable investigation based on the self supplied gate driver powering solution presented already in [13]. The investigation results will be compared in the last section of the paper based on effectiveness, integrability and ease of implementation.

\section{SUPPLY AND DRIVING TOPOLOGIES}

\section{A. Gate driver circuits}

Gate driver topologies can be classified as a function of the supplies they are associated with. Basically, the supply is either unipolar or bipolar. Bipolar gate driver signals are usually preferred because they offer better turn OFF dynamics as well as a natural and effective EMI shielding inside the power devices. Therefore, even if needed performances and operational behavior can be reached with 
unipolar gate drivers, we focus our investigations on the solutions that can generate and apply bipolar control signals to the gate capacitance of the power devices. Considering the association of the gate driver with a bipolar supply, a simple push-pull circuit is enough to drive the power switch with bipolar signals. The topology of the gate drivers is given on Figure 1. With such a topology, the gate capacitance can be driven from the voltage sources but accurate current feeding can also be implemented. This allows the driver to control the gate charge and to shape the switching transition.

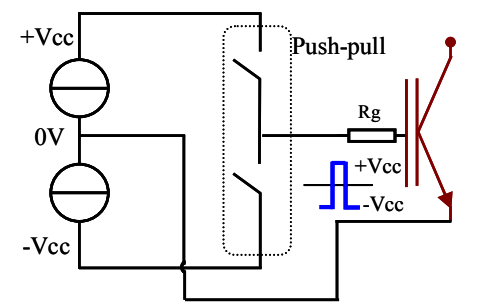

Figure 1. Bipolar gate driver consist of bipolar supply and push-pull topology

In this case, the gate capacitance is fed from the positive supply and then discharged applying the negative source between gate and source (or emitter). Hence, the energy stored in the gate capacitance is dissipated in the negative source each switching cycle. The power consumption of the gate driver is then a function of the gate charge, the switching frequency and the gate driver's supply voltage. At reasonable switching frequencies (between $10 \mathrm{kHz}$ and $50 \mathrm{kHz}$ ), the power consumption of the gate drivers remains small and the gate driver supplies may be implemented without trouble since their efficiency level is not critical. As an example, let us consider a practical case with a well known power device, the IRF740 having a gate capacitance of $63 \mathrm{nC}$ driven at $35 \mathrm{kHz}$, under $+15 /-15 \mathrm{~V}$ and supplied by sources having $30 \%$ efficiencies. The total gate driver's power consumption is in the range of $0.5 \mathrm{~W}[3]$ knowing that this kind of device is usually used to convert electric power in the range of 500 up to $1 \mathrm{~kW}$. The gate signal can be shaped as needed if the push pull structure is operated in current mode. A simple implementation is depicted on Figure 2. If possible, the negative supply can be lowered to $-5 \mathrm{~V}$ which is usually enough to enable current control and natural shielding. This improves slightly the gate driver's power consumption.

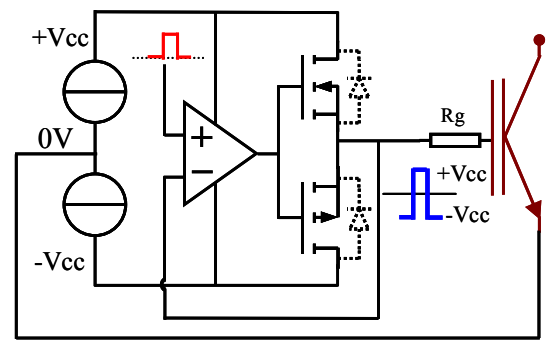

Figure 2. Bipolar gate driver with bipolar supply and current amplifier topology
At higher switching frequencies and for larger power devices, the gate driver's power consumption may become excessive and it requires either the implementation of effective gate driver supplies or a drastic reduction in the consumption levels of the gate drivers. Efficient gate driver supplies will be costly and will require specific designs. They are much harder to integrate and lead to increased failure risk for the whole converter. On the other hand, more efficient driving solutions for high frequency operation have been studied based on regular bipolar supplies and push pull structure [14]. Figure 3 presents one of these solutions where the push pull structure is associated to a resonant topology that "recycles" part of the energy withdrawn from the gate capacitance each switching transition.
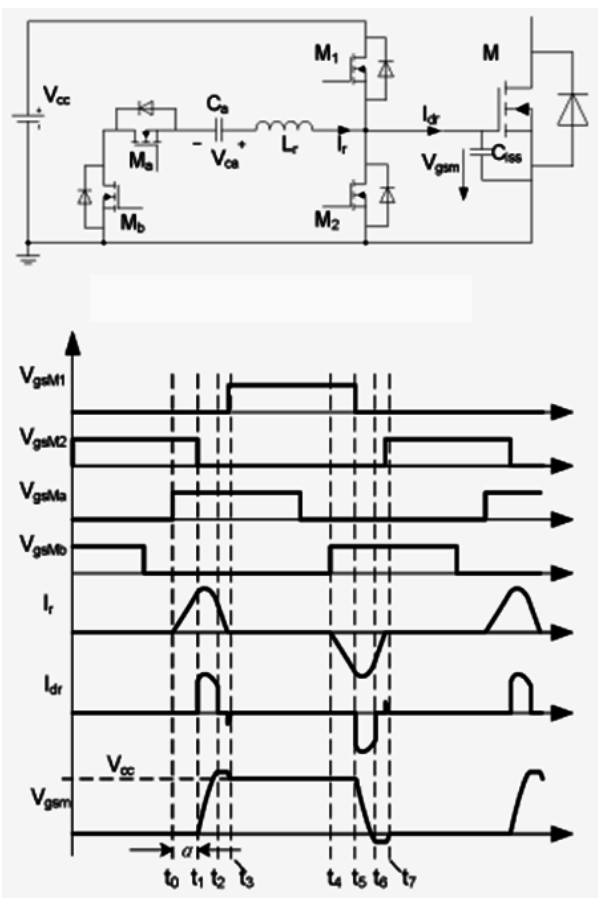

Figure 3. Resonance MOSFET driver circuit [14]

This structure allows the gate driver to save a large part of the energy needed in order to charge or to discharge the gate capacitance of the power device. Thus, it lowers the power needed and decreases the requirements on the power supply while improving the overall efficiency of the power converter.

If a unipolar supply is considered, the topology of the gate driver must be modified. At first, a second voltage supply can be generated from the first one with the help of a buck-boost chopper. This structure is described in Figure 4.

Another solution consists in using a resonant LC structure to discharge the gate capacitance and to invert the voltage across the gate to source terminals. This gate driver topology is shown on Figure 5. Theoretically, half of the energy consumption is saved in this case and a negative potential is applied to the gate terminals during the OFF 
state. However, the dynamics are limited by the value of the LC circuit or the pulse discharge current making this solution either difficult to integrated or possibly susceptible to external parasitic.

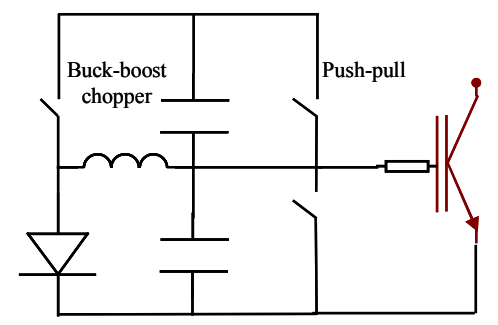

Figure 4. Unipolar supply associative with buck-boost chopper and push pull topology

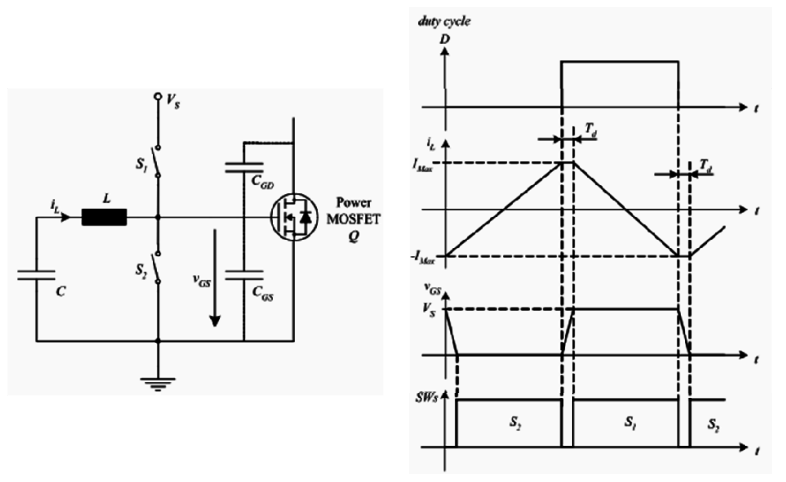

Figure 5. Resonant gate driver[15]

The most flexible and effective solution relies on the implementation of a full bridge converter to drive the gate capacitance. In this configuration, one inverter harm middle point is connected to the gate terminal whereas the other inverter harm middle point is connected to the source terminal of the power device. The DC + and DC- terminals are connected to the plus and minus terminals of the unipolar gate driver source. Hence, it is possible to implement resonant converter structure in order to optimize the gate driver power consumption. The structure can also be used to drive the current to the gate, suitable for precise and specific gate charge evolution. Figure 6 below presents the topology in case of a regular implementation. It can be noticed that the floating source voltage reference is not anymore tied to the power device voltage reference i.e. the source terminal in case of a power MOSFET or the emitter terminal in case of an IGBT. This topology is of interest thanks to its monolithic integration possibility and reduced number of passive components (only one storage capacitor).

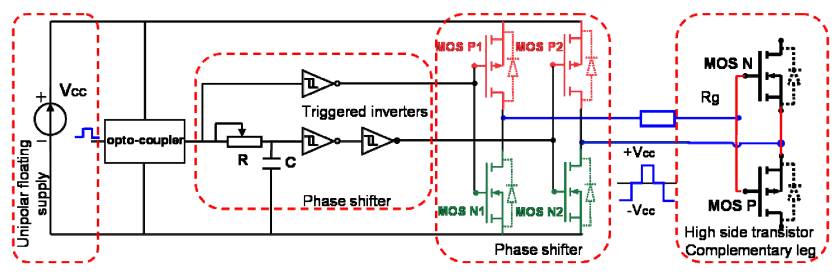

Figure 6. Unipolar supply associative with full bridge converter that drives a complementary inverter leg

This ends our brief review of the main gate driver topologies that can be used to apply bipolar gate signals under high frequency operation and depending on the gate supply types. We focus now on the gate driver supplies with a short overview of the main topologies.

\section{B. Isolated gate driver power supplies}

High side power devices or complementary P-N structures [12] require an isolated floating voltage source that can be referenced to the bottom terminal of the power device, which needs to be driven. Basically, we identify four approaches that are regularly used or presented in the literature. The most basic one is the linear regulator which is simple to implement and exhibits extremely low efficiency levels. The energy is usually taken from the power side through a resistor and regulated thanks to an avalanche diode. The second one is the regular DC to DC isolated converter using a $\mathrm{HF}$ or piezoelectric transformer [16]. Numerous studies have been carried out on the design and the optimization of such small power supplies [17]. Recently, several research works are related to the integration of these low voltage and low power converters that still offer high voltage isolation levels. We can cite a commercially available device [18] and research works $[19][20][21][22]$. These supplies can be duplicated to implement bipolar supplies, more suitable for bipolar gate signal generation. However, these integrated converters have limited efficiency levels of $33 \%$ for the industrial ones and around 50 to $60 \%$ for the lab demonstrators. Figure 7 depicts the topology of such converter and a possible 3D integrated assembly. Isolated supplies based on discrete components can be more efficient but require specific designs [23]. Besides, their robustness may affect the global reliability of the converter if it is not integrated.

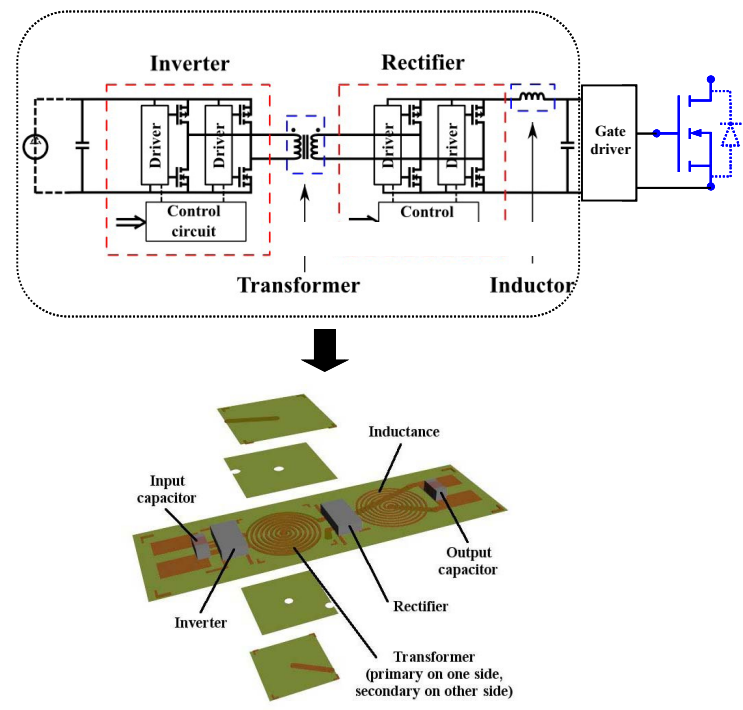

Figure 7. Integrated isolated DC/DC micro-converter[19]. 
Another popular driver supply is the bootstrap topology that takes advantage of the converter operation to charge up periodically a capacitor tank, which is used to supply the floating gate driver of the high side power devices. The topology of the bootstrap technique is given on Figure 8. Each time the bottom power switch is conducting, a circuit formed by the bootstrap diode, the storage capacitor, the low side gate driver supply and the bottom device allowing the recharge of the storage device. When the bottom switch is OFF, the bootstrap diode is blocked and the high side gate driver is supplied thanks to the energy stored in the storage capacitor Cs. This requires periodical recharge sequences and therefore permanent converter operation. The classical bootstrap technique corresponds to a unipolar supply.

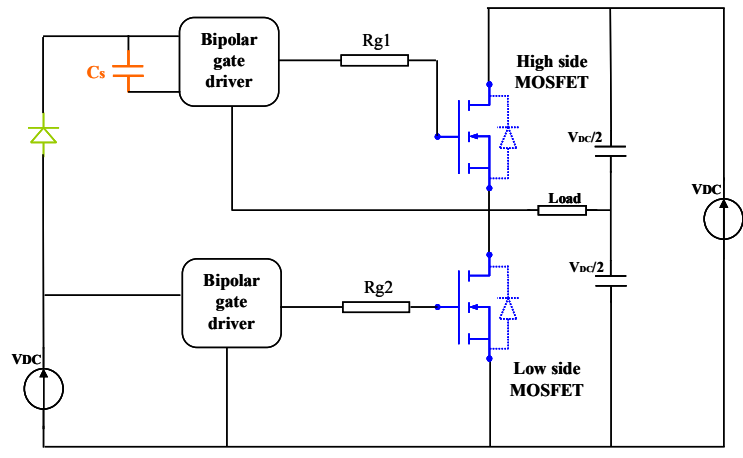

Figure 8. Conventional bootstrap technique topology

This bootstrap technique can be improved as it is presented in [7]. The first improvement is a self controlled bootstrap that operates independently to the power side, simplifying the design requirement and removing the possible operational limits. An extension is proposed in [7] in order to "emulate" a bipolar driver supply based on the unipolar topology. Although this approach requires a large extend in component number, it seems to offer a possible solution. Its topology is presented on Figure 9(a)

Another solution is patented on an extension of the unipolar structure in order to derive a bipolar structure. Figure 9(b) below shows its topology. The approach is similar to the classical topology exception made to the additional switch which is required to open the negative supply loop while the bottom switch is turned ON.

The last topology considered here is the self-supply technique that is implemented across the power device to be driven. The classical topology of this technique is depicted on Figure 10. This supply is also based on a periodical recharge of a storage capacitor and a pulse linear regulation approach [24]. Thanks to this particular operation, the efficiency of the supply can be optimized while being simple to integrate. This gate driver supply is unipolar type and can be extended to bipolar type thanks to a symmetric structure depicted on Figure 11.

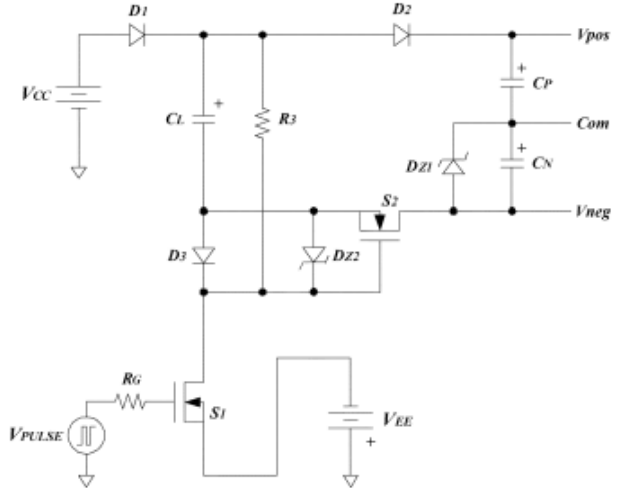

(a)

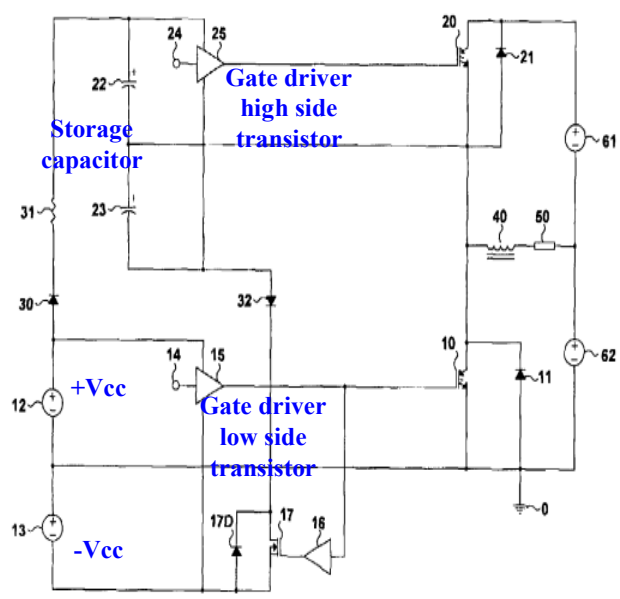

(b)

Figure 9. (a) Self-boost charge pump topology with positive- and negative-bias voltage supply capability [7]. (b) Boostrap technique with bipolar supply[6]

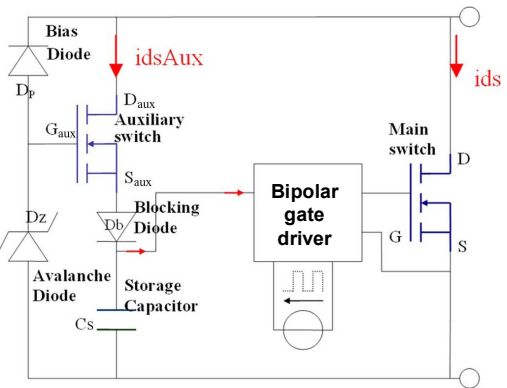

(a)

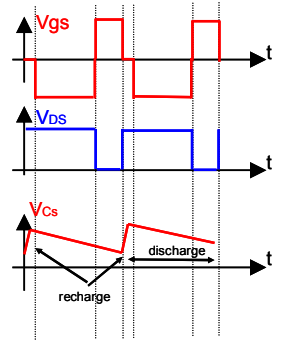

(b)
Figure 10. Self-supply technique topology [24] 


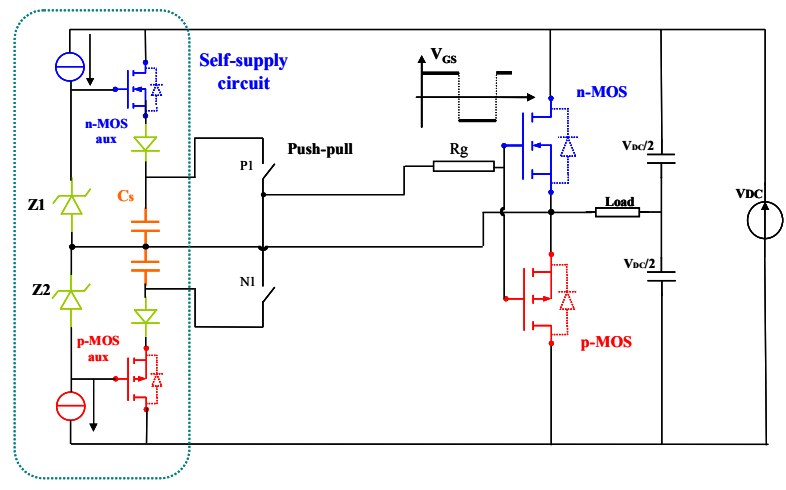

Figure 11. Symetric topology of bipolar self-supply technique

\section{DRIVERS PLUS BOOTSTRAP POWER SUPPLIES}

The voltage regular bootstrap technique provides a floating unipolar source to the high side switch gate driver. Based on the overview presented in the previous section, this type of supply must be added to specific gate driver topologies in order to control the power switch with bipolar gate signals. The other approach consists in using the bipolar bootstrap supply plus a push pull gate driver structure. In all cases, a major limitation of this approach for high frequency operations may remain the fact that a minimum time is required to recharge the storage capacitor each switching cycle, leading to a minimum low side switch duty cycle and leading to a limited maximum switching frequency.

\section{A. Bipolar bootstrap plus push-pull topology}

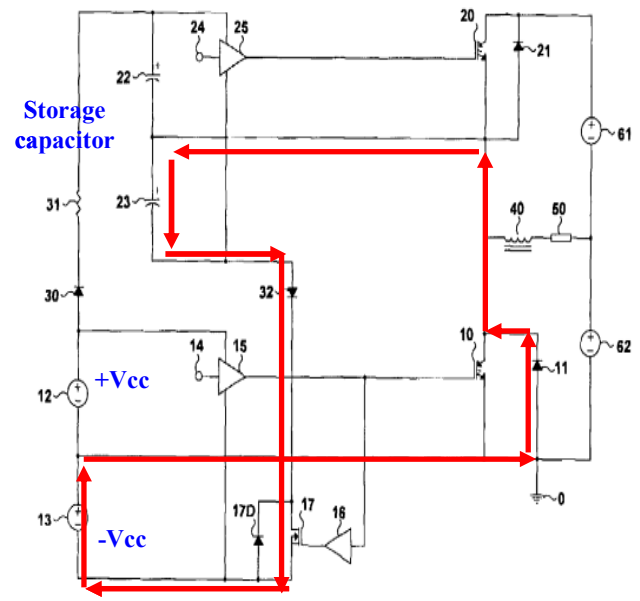

Figure 12. Bipolar Boostrap technique with negative recharge loop[6]

The topology of the bipolar bootstrap technique is presented in Figure 9. The bipolar supply is composed of two unipolar bootstrap supplies exception made the addition of a high voltage switch inserted in series with the negative bootstrap loop. It is required in order to prevent current flowing from the power side down to the negative gate driver supplies while the high side switch is in its ON state. It is controlled with the same control signal as the one fed to the low side power switch gate driver keeping the global control of the structure simple. However, the added high voltage switch needs to maintain opend the negative bootstrap loop when needed that remains not simple to drive, because it is also at a floating potential making this solution not simple to implement from a hardware point of view (Figure 12). Nevertheless, this gate driver supply can be associated with a push pull topology in order to perform bipolar control signals to the high side switch.

\section{B. Unipolar bootstrap plus full bridges}

Considering unipolar bootstrap technique to supply a gate driver that must perform bipolar gate signals, resonant gate drivers may be a solution. A combination of this type of driver supply plus the resonant circuit presented in Figure 5 is straightforward. It may minimize the implementation cost and complexity exception made for the required passive devices. A structure that is more complex could be considered. As an example, the combination of the unipolar bootstrap technique plus a full bridge gate drive inverter could be interesting. Figure 13 below presents the full topology of this approach.

However, a precise analysis of the global behavior shows that this is not as straightforward as it seems. In a similar manner as in the previous section, the combination of these two topologies ends up offering a leakage current path between the power circuit and the gate driver. This is detailed on Figure 13 with the visualization of the fault current path when the top switch is turned OFF.

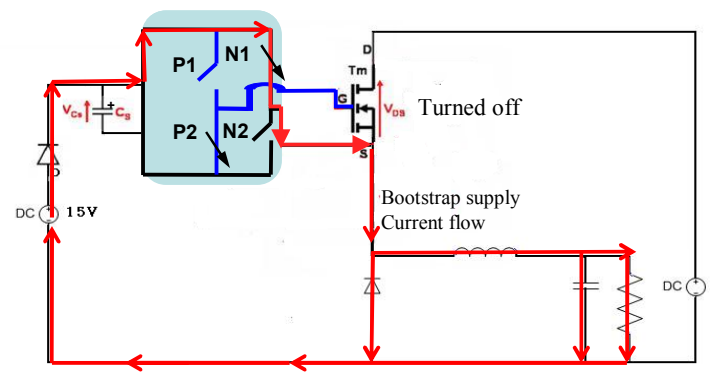

Figure 13. Bootstrap supply technique associative with full bridge gate driver
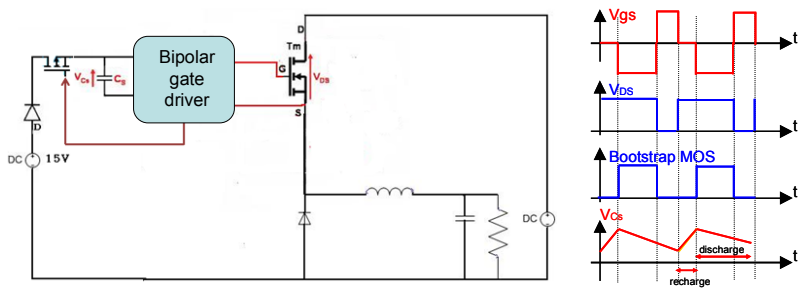

Figure 14. Boostrap bipolar supply with additional current limiting transistor

In a similar manner, as in part $\mathrm{A}$, it is therefore required to insert a transistor in the fault current loop in order to open the loop when needed (Figure 14). Preferably, this switch can be a $\mathrm{P}$ type transistor that must be turned OFF when the high side transistor is turned ON. It does not increase too much the complexity of the structure may be 
monolithically integrated within the gate driver circuit die. Indeed, it is a low voltage device rating.

\section{Limits of these approaches}

The unipolar bootstrap supply technique is already known for its ease of implementation and operation. It is also well known for its operational limitations due to its interactions with the power parts. If solutions are provided to emulate autonomous operation and bipolar supply [7][6], these gate driver supply solutions which rely on numerous components are not always simple to integrate. This leads to an increase in cost, design and a possible reduction of reliability making these approaches not always competitive with integrated DC to DC supplies such as [18].

\section{DRIVERS PLUS SELF SUPPLY TECHNIQUE}

Another high side gate driver supply technique that can be considered is the self powering technique presented in Figure 10. This type of unipolar power supply can be integrated without extra cost. Its evolution and combination with a haddock gate driver to perform bipolar gate signals is now going to be investigated. If the association with resonant gate drivers such as those presented in figure 5 and 6 is more or less straightforward, the association with the full bridge gate driver is more complex.

\section{A. Unipolar self supply plus full bridge gate driver}

The unipolar self-powering technique is based on the pulsed linear regulation technique that periodically, picks up some energy from the power side at each high side transistor turn OFF [25]. The self-supply is set to be sensitive only to positive $\mathrm{dv} / \mathrm{dt}$ and recycles a part of commutation energy to supply the gate driver. Basically, a path is temporary offered for the load current in parallel with the switch which is turning OFF. This path includes the gate driver storage capacitor. Once it is recharged, the path is closed and the switching transition is quickly terminated [25]. Based on this operation, the addition of a full bridge gate driver is not as straightforward as it seems. Indeed, while the high side power device is turned OFF by the bipolar commutation of the full bridge gate driver, an attention must be paid in order to maintain available the path for the load current to flow through the storage capacitor. The figure 15 below presents the full topology including the gate driver and the driver supply. It is shown that during the turn OFF sequence, the bridge harm connected to the source terminal of the power transistor must be at first maintained into a specific configuration before switching in order to apply the correct voltage level to the gate to source terminals of the power switch to drive. Therefore, the full bridge converter must operate under phase shift control in order to be correctly combined with the self power supply technique. Figure 16 depicts simulation results showing this particular switching transition procedure.

This approach has been implemented on a PCB in order to test and to validate this functionality. Figure 17 below gives a set of practical results demonstrating the operation of the whole gate driver. It appears that the power flow through the self supply technique is in the range of $1 \mathrm{~W}$, including its own efficiency. This quite large power consumption could be minimized if resonant gate drivers were implemented.

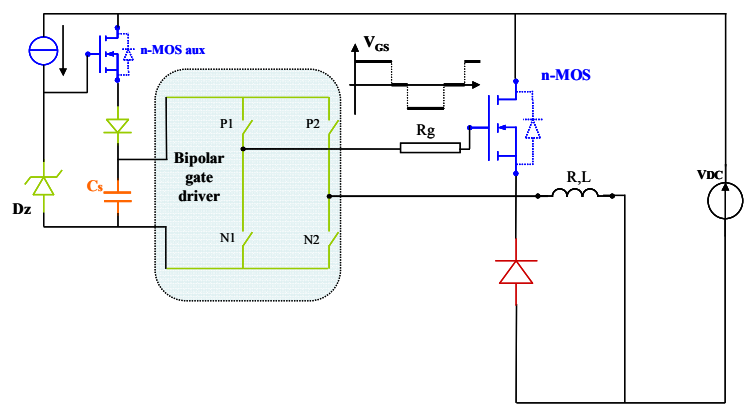

Figure 15. Self-supply in combination with full bridge gate driver

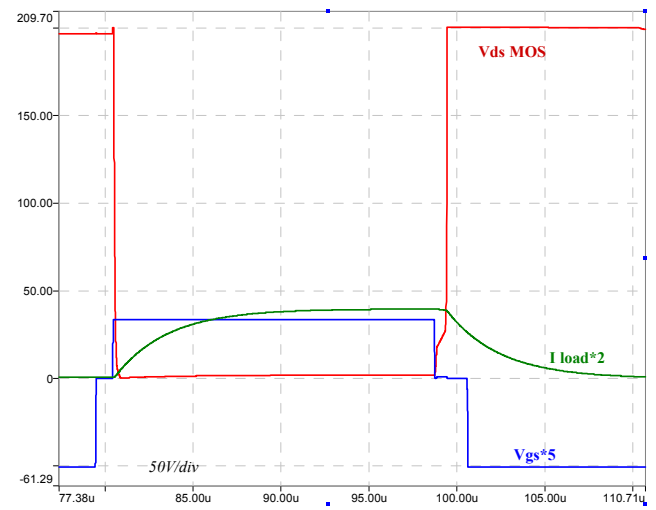

Figure 16. Simulation results of a Buck converter with full gate driver topology

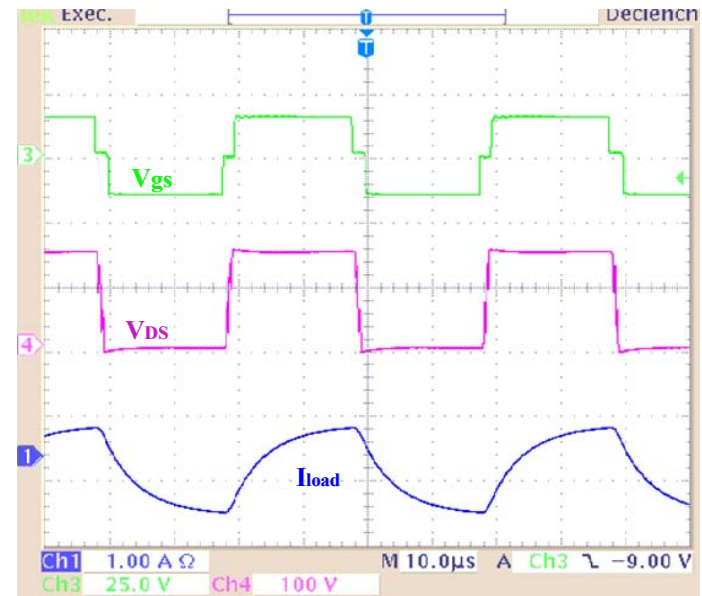

Figure 17. Pratical results of a half-bridge inverter with full gate driver topology

Besides, the phase shift control of the gate bridge may appear less flexible to manage the turn ON turn OFF of the power device since the three levels operation does not 
allow to pull back the gate current as desired. One way to optimize its effect is to monitor the recharge sequence of the storage capacitor in order to minimize the zero level duration and to keep switching dynamics at their best levels. This can be implemented based on the schematic presented on Figure 15. In this case, the switching transition of the second harm of the driver bridge is engaged only but also shortly after the capacitor has been recharged.

Nevertheless, this approach remains highly integrable and may offer satisfactory operation from both efficiency and dynamics point of views.

\section{B. Bipolar self supply plus push pull gate driver}

The evolution of the self supplied technique can also be investigated. It is based on a symmetrical supply technique trying to take advantage of the two switching transitions that occur each switching period of a commutation cell. The resulting topology was depicted on Figure 11. This topology remains sensitive only to positive $\mathrm{dv} / \mathrm{dt}$ but this time coming from both active power devices. The driver supply structure is more complex but the gate driver implementation can be simplified to a push pull structure including or not a resonant structure in order to save on power consumption. Figure 18 shows experimental results coming from this topology.

The only bad points of this structure are that it is harder to integrate since two storage capacitors are required. In addition, the most critical point must consider that the load current does not switch from positive to negative within each switching cycle in all applications. In this way, one of the driver supply (the positive one if the load current enter the middle point and the negative supply if the load current exits the middle point, as shown on Figure 18) will not be fed periodically by the load current but by the power converter supply. Therefore, the efficiency of such gate driver supply may be largely reduced limiting the operation of the structure to low power, low to medium voltage applications.

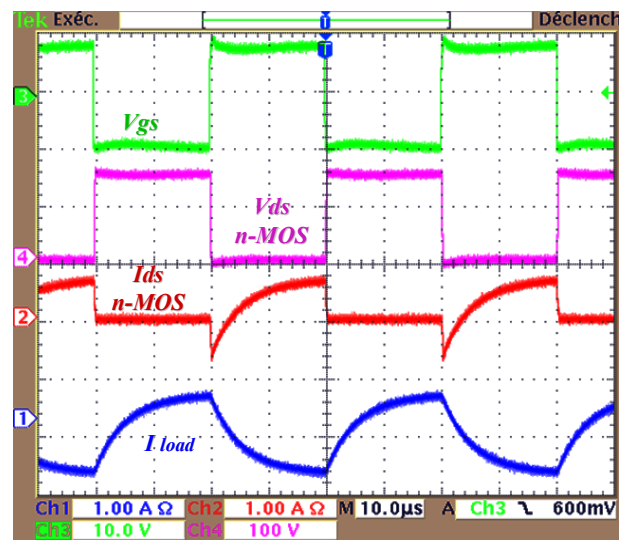

Figure 18. Experimental results of bipolar self supply associative with push pull gate driver

\section{Symetrical unipolar self supply plus full bridge.}

In order to overcome these limitations, a last configuration can be considered. It relies on the topology Figure 19 where the bipolar gate driver self supply has been merged to a combined structure where only one capacitor remains. This capacitor can be recharged periodically by both driver supplies or only by one of them as a function of converter operation. In this case, the three level driver bridge operation is required but the gate driver supply operation can be optimized. Its integration remains possible if complementary devices are used. Below Figure 20 is given a set of simulation results.

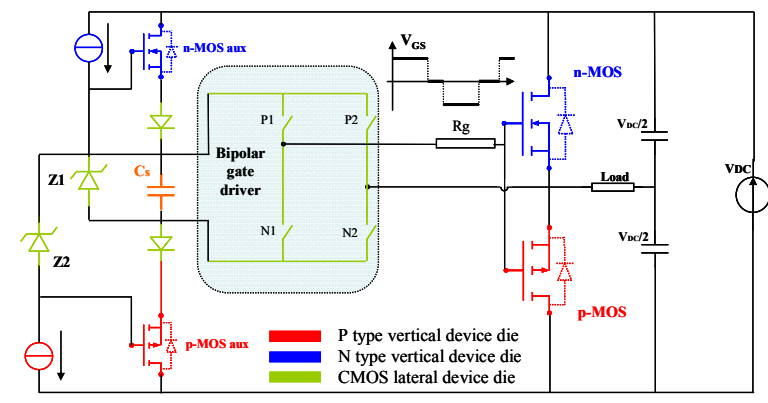

Figure 19. Symetrical unipolar self supply with full bridge gate driver topology

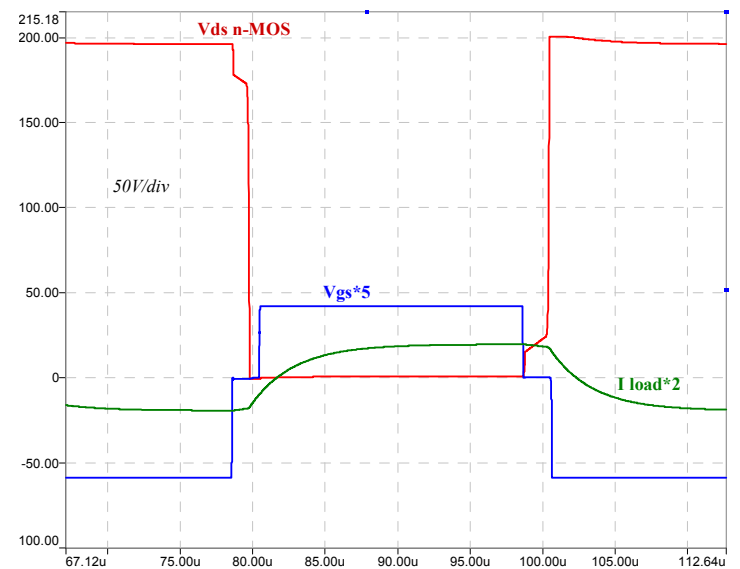

Figure 20. Simulation results of symetrical self supply topology with gate driver

\section{CONCLUSION}

The paper presents an overview of possible combination of gate drivers and driver supplies for the implementation of bipolar control gate signals. It appears that simple and flexible unipolar gate driver powering techniques are not always simple to emulate toward bipolar supplies. This leads to more complex schematics and numerous additional components not always simple to integrate. Nevertheless, it appears that:

- The bootstrap technique can be modified to implement bipolar gate driver supply and selfoperation. Associated with resonant gate driver circuits, it may be a good solution for medium to 
high power application and high switching frequencies. The solutions remain hard to integrate.

- The self-powering supply technique can be modified to implement bipolar gate driver supply. The solutions are quite simple to integrate but their efficiency and dynamic behavior may become a limitation in some cases.

The following steps must now consider deeply the efficiency and dynamic characterization of the solutions, introducing the resonant topology in the gate drivers to optimize the operation of these circuits. On the other hand, the integration remains also an issue in order to maintain the robustness of the global converter.

\section{REFERENCES}

[1] Zhihua Yang, Sheng Ye, et Yan-Fei Liu, "A New Dual-Channel Resonant Gate Drive Circuit for Low Gate Drive Loss and Low Switching Loss," Power Electronics, IEEE Transactions on, vol. 23, 2008, pp. 1574-1583.

[2] Zhiliang Zhang, W. Eberle, Ping Lin, Yan-Fei Liu, et P. Sen, “A new hybrid gate drive scheme for high frequency buck voltage regulators," Power Electronics Specialists Conference, 2008. PESC 2008. IEEE, 2008, pp. 2498-2504.

[3] J. Strydom, M. de Rooij, et J. van Wyk, "A comparison of fundamental gate-driver topologies for high frequency applications," Applied Power Electronics Conference and Exposition, 2004. APEC '04. Nineteenth Annual IEEE, 2004, pp. 1045-1052 vol.2.

[4] R. Pilawa-Podgurski, A. Sagneri, J. Rivas, D. Anderson, et D. Perreault, "Very-High-Frequency Resonant Boost Converters," Power Electronics, IEEE Transactions on, vol. 24, 2009, pp. 1654-1665.

[5] C. Klumpner et N. Shattock, "A Cost-Effective Solution to Power the Gate Drivers of Multilevel Inverters using the Bootstrap Power Supply Technique," Applied Power Electronics Conference and Exposition, 2009. APEC 2009. Twenty-Fourth Annual IEEE, 2009, pp. 1773-1779.

[6] Rick West, "Bipolar boostrap top switch gate driver for half-bridge semiconductor power topologies," U.S. Patent US7248093.

[7] Shihong Park et T. Jahns, "A self-boost charge pump topology for a gate drive high-side power supply," Power Electronics, IEEE Transactions on, vol. 20, 2005, pp. 300-307.

[8] N. Rouger et J. Crebier, "Toward Generic Fully IntegratedGate Driver Power Supplies," Power Electronics, IEEE Transactions on, vol. 23, 2008, pp. 2106-2114.

[9] B. Nguyen, J. Crebier, R. Mitova, L. Aubard, et C. Schaeffer, "AC switches with integrated gate driver supplies," Power Electronics and Applications, 2005 European Conference on, 2005, pp. 9 pp.P.9.
[10] Baoxing Chen, "Isolated half-bridge gate driver with integrated high-side supply," Power Electronics Specialists Conference, 2008. PESC 2008. IEEE, 2008, pp. 3615-3618.

[11] Manh Hung Tran et J. Crebier, "Complementary MOS structures for common mode EMI reduction," Power Electronics and Applications, 2009. EPE '09. 13th European Conference on, 2009, pp. 1-10.

[12] M.H. Tran, J. Crebier, et C. Schaeffer, "Quantification of benefits and drawbacks in power conversion based on complementary MOS structures," Energy Conversion Congress and Exposition, 2009. ECCE. IEEE, 2009, pp. 3423-3430.

[13] N. Rouger, J. Crebier, R. Mitova, L. Aubard, et C. Schaeffer, "Fully integrated driver power supply for insulated gate transistors," Power Semiconductor Devices and IC's, 2006 IEEE International Symposium on, 2006, pp. 1-4.

[14] S. Pan et P. Jain, "A New Pulse Resonant MOSFET Gate Driver with Efficient Energy Recovery," Power Electronics Specialists Conference, 2006. PESC '06. 37th IEEE, 2006, pp. 1-5.

[15] T. Lopez, G. Sauerlaender, T. Duerbaum, et T. Tolle, "A detailed analysis of a resonant gate driver for PWM applications," Applied Power Electronics Conference and Exposition, 2003. APEC '03. Eighteenth Annual IEEE, 2003, pp. 873-878 vol.2.

[16] D. Vasic, F. Costa, et E. Sarraute, "Piezoelectric transformer for integrated MOSFET and IGBT gate driver," Power Electronics, IEEE Transactions on, vol. 21, 2006, pp. 56-65.

[17] S.P. Vlahu, "High frequency pulse transformer for an IGBT gate drive ," U.S. Patent USRE38082, Avril 22, 2003.

[18] B. Chen, "Fully integrated isolated DC-to-DC converter and half bridge gate driver with integral power supply," Cork, Ireland, 2008: .

[19] O. Deleage, J. Crebier, M. Brunet, Y. Lembeye, et H. Tran Manh, "Design and realization of highly integrated isolated DC/DC micro-converter," Energy Conversion Congress and Exposition, 2009. ECCE. IEEE, 2009, pp. 3690-3697.

[20] J. Popovic et J. Ferreira, "Design and evaluation of highly integrated dc-dc converters for automotive applications," Industry Applications Conference, 2005. Fourtieth IAS Annual Meeting. Conference Record of the 2005, 2005, pp. 1152-1159 Vol. 2.

[21] Z. Hayashi, Y. Katayama, M. Edo, et H. Nishio, "High efficiency DC-DC converter chip size module with integrated soft ferrite," Magnetics Conference, 2003. INTERMAG 2003. IEEE International, 2003, pp. FC-04.

[22] V. Kursun, S. Narendra, V. De, et E. Friedman, "High input voltage step-down DC-DC converters for integration in a low voltage CMOS process," Quality Electronic Design, 2004. Proceedings. 5th International Symposium on, 2004, pp. 517-521.

[23] J. Popovic et J. Ferreira, "Converter concepts to increase the integration level," Power Electronics, IEEE Transactions on, vol. 20, 2005, pp. 558-565.

[24] N. Rouger, J. Crebier, et S. Catellani, "High-Efficiency and Fully Integrated Self-Powering Technique for Intelligent Switch-Based Flyback Converters," Industry Applications, IEEE Transactions on, vol. 44, 2008, pp. 826-835.

[25] J. Crebier et N. Rouger, "Loss Free Gate Driver Unipolar Power Supply for High Side Power Transistors," Power Electronics, IEEE Transactions on, vol. 23, 2008, pp. 1565-1573. 